\title{
Intelligent agents in biomedical engineering: a systematic review
}

\begin{abstract}
The design and implementation of Multi Agent System (MAS) has gained more attention over the last 10 years due to the advance of artificial intelligence, wireless sensor network and microelectronic devices for the Internet of Things (IoT). IoT has ceased to be a novelty, becoming a reality in people's daily lives, since wearable devices and smartphones have become a daily basis tool for a large part of the population. The level of resolution and detection of problems have become a bottleneck for such technologies, which the human error is not more admissible. Since heterogeneity and autonomy are desirable characteristics in IoT systems, especially when such systems are aimed at monitoring the health of their user, MAS is highly required. This research provides a systematic review on the use of MAS in biomedical engineering, focusing on interactions in healthcare environments, architecture and inter-agent communication.
\end{abstract}

Keywords: intelligent agents, artificial intelligence, internet of things, biomedical engineering, healthcare
Volume 6 Issue 5 - 2020

\section{Tatiana Pereira Filgueiras, Pedro Bertemes- Filho \\ Department of Electrical Engineering, State University of Santa} Catarina, Brazil

\author{
Correspondence: Pedro Bertemes Filho, Department of \\ Electrical Engineering, State University of Santa Catarina, Rua \\ Paulo Malschitzki 200, Zona Industrial Norte, Joinville, Santa \\ Catarina, Brazil, Tel +55(47)348I7848, \\ Email pedro.beremes@udesc.br
}

Received: December 18, 2020 | Published: December 28, 2020

\section{Introduction}

With the rise and development of technology, it is necessary to improve the way that data is collected and treated, aiming to better the intelligence in the process and also helping in the formatting, treatment and decision making regarding the collected information. Distributed Artificial Intelligence (DAI) is the system class that allows for various processes called agents to interact, distributing themselves logically or spatially, being autonomous and smart; it is also the intersection between Distributed Computing and Artificial Intelligence (AI). ${ }^{1}$ From an architectural point of view, intelligent agents (IAg) are entities composed of a unique identifier and three main components: code, data and status, in addition to having a life cycle associated with their state of execution. ${ }^{1,2}$ In a Multi Agent System (MAS), it is possible to delegate different tasks to different agents, where a larger problem is divided into small sub problems, and each agent produces an output in accordance with its mission (task), where, later, all outputs are joined and converted into the final answer to the total problem. ${ }^{1-4}$ In addition, agents can interact with each other and share common data in order to increase the speed of problem resolution. As for the characteristics, a given IAg can be classified according to the system architecture (Open or Closed Code), the knowledge representation, its mobility (Static and Mobile Agents), its intelligence mechanisms (AI, ontology, etc.), the interaction/communication (basic, passive, active and interlocutor), the system's complexity, scalability and security, and privacy. ${ }^{2-4}$

Static (or stationary) agents can only be executed on the machine they were started on, and cannot perform tasks remotely, even though they have the ability to interact with remote agents. ${ }^{1,5}$ Mobile agents are those which can migrate from one device to another, taking data and status with them, being able to perform tasks locally and remotely. Mobile agents can migrate between MAS hosts to use their resources locally. ${ }^{1,5}$ The scalability of a MAS can be defined by the number of agents, amount of communication, number of tasks and amount of resources that can be increased or reduced in execution time without affecting the system. ${ }^{2,6}$ Knowledge representation in a MAS is different from other computer systems, since they have reasoning mechanisms and autonomous distributed interactions, not only dealing with static information, but also dynamic. ${ }^{1,3,4,6}$ Communication between agents of a MAS (inter-agent) takes place through the use of pre-defined protocols or programming languages..$^{1,2}$ IAgs are heterogeneous, distributed and autonomous models, which can be easily compared and mapped in the health care domain, since this domain has many distinct pathologies and care strategies, in addition to the heterogeneity of patients, also allowing for the creation of personalized models of care. ${ }^{1,2,6}$ The use of MAS in the context of health can assist in a quick and intelligent way the decision making in a certain case, optimizing the patient care process, if necessary. ${ }^{3-6}$ Since the components of a healthcare system and the ones involved in it can be considered agents, and that the interaction between parts of the system and between systems is also possible, both in solving a problem and in assisting in decision-making, ${ }^{6,7}$ the use of MAS in Biomedical Engineering (BE) becomes attractive. Some solutions have been proposed by the academic community, such as fall detection, ${ }^{7}$ Home Care of bedridden and/or elderly patients ${ }^{7-10}$ and continuous monitoring of vital signs for later use by the physician. ${ }^{8,11}$ The objective of this research is to investigate practical cases of intelligent agents and MAS applications in biomedical engineering, focusing on the interactions in healthcare environments and the how they operate regarding the data architecture used and the inter-agent communication.

\section{Material and methods}

Literature searches were carried out regarding DAI, IAg and MAS applied in Biomedical Engineering. The study was based on scientific works published in these expertise areas. A big search was performed in the ACM,12 IEEE13 and Science Direct databases.14 Some of them were made using filters such as publication date and keywords while others, only the keywords (Table 1). The first six publications that appeared as the first search results were selected. These address the use of MAS and the collection of vital signal data from a particular group of volunteers. These surveys cover a period from 2008 to 2020 and the keyword used was "health monitoring" multi-agent system. 
Table I Number of articles and papers published from 2008 to 2020 according to the selected keywords of interest

\begin{tabular}{|c|c|c|c|c|}
\hline Search & Database & Keyword & Date range & Results \\
\hline I & IEEE & health Multi Agent system & no filter & 25.900 \\
\hline 2 & IEEE & health Multi Agent system & $2008-2020$ & 22.100 \\
\hline 3 & ACM & health Multi Agent system & no filter & 5.180 \\
\hline 4 & $\mathrm{ACM}$ & health Multi Agent system & $2008-2020$ & 4.430 \\
\hline 5 & Science Direct & health Multi Agent system & no filter & 180.000 \\
\hline 6 & Science Direct & health Multi Agent system & $2008-2020$ & 149.000 \\
\hline 7 & IEEE & Multi agent system health engineering & no filter & 72.900 \\
\hline 8 & IEEE & Multi agent system health engineering & $2008-2020$ & 56.500 \\
\hline 9 & ACM & Multi agent system health engineering & no filter & 11.300 \\
\hline 10 & ACM & Multi agent system health engineering & $2008-2020$ & 8.170 \\
\hline II & Science Direct & Multi agent system health engineering & no filter & 172.000 \\
\hline 12 & Science Direct & Multi agent system health engineering & $2008-2020$ & 142.000 \\
\hline 13 & IEEE & Multi Agent Health Care & no filter & 5.050 \\
\hline 14 & IEEE & Multi Agent Health Care & $2008-2020$ & 4.460 \\
\hline 15 & $\mathrm{ACM}$ & Multi Agent Health Care & no filter & 920 \\
\hline 16 & ACM & Multi Agent Health Care & $2008-2020$ & 800 \\
\hline 17 & Science Direct & Multi Agent Health Care & no filter & 4.380 \\
\hline 18 & Science Direct & Multi Agent Health Care & $2008-2020$ & 3.770 \\
\hline 19 & IEEE & "health monitoring" multi-agent system & no filter & 927 \\
\hline 20 & IEEE & "health monitoring" multi-agent system & $2008-2020$ & 857 \\
\hline 21 & ACM & "health monitoring" multi-agent system & no filter & 86 \\
\hline 22 & $\mathrm{ACM}$ & "health monitoring" multi-agent system & $2008-2020$ & 72 \\
\hline 23 & Science Direct & "health monitoring" multi-agent system & no filter & 440 \\
\hline 24 & Science Direct & "health monitoring" multi-agent system & $2008-2020$ & 427 \\
\hline
\end{tabular}

\section{Results}

\section{Data analysis}

As a result by the literature review, it was narrow down the main characteristics of those researches, as shown in Tables $2 \&$ Tables 3 . As for the purpose of the studies' proposals, some suggest an architectural model for the further development of a solution for monitoring health using MAS Design and Development) MADIP, ${ }^{8}$ MASRHMS, ${ }^{9}$ MBES $^{15}$ and MEMMHCS ${ }^{11}$ while others suggest the solution already implemented (System) such as MADIP ${ }^{8}$ and DDMmS. ${ }^{10}$ With regard to the target population, there are those researches that focus especially on the elderly public MADIP ${ }^{8}$ and $\mathrm{DDMmS}^{10}$ and the bedridden one MADIP, ${ }^{8}$ MADRHMS, ${ }^{9}$ while others are recommended to the general public MBES ${ }^{15}$ and MEMMHCS. ${ }^{11}$ Most studies have one or more devices, which measure different parameters from the volunteer (Operation). Some of these devices are used for remote patient care MADIP, ${ }^{8}$ MASRHMS,${ }^{9}$ DDMmS, ${ }^{10}$ and MEMMHCS ${ }^{11}$ while others are used for hospital care using hospital-specific devices MADIP ${ }^{8}$ and MBES. ${ }^{15}$ It was noticed that the technology used by most publications is wearable, such as: MADIP, ${ }^{8}$ MASRHMS, ${ }^{9} \mathrm{MBES}^{15}$ and DDMmS. ${ }^{10}$ Others include the use of smartphone and other mobile devices: MADIP ${ }^{8}$ and MEMMHCS, ${ }^{11}$ and there are still those that mix the use of all the aforementioned technology: MADIP. ${ }^{8}$ 
Table 2 Case of study published from 2008 to 2020 according to the objective and target population

\begin{tabular}{llll}
\hline Case study & Year & Objective & Target population \\
\hline MADIP & 2008 & Design and Development & Bedridden patients \\
MASRFid & 2011 & System & The elderly \\
MASRHMS & 2016 & Design and Development & The elderly and bedridden patients \\
MBES & 2017 & Design & General population \\
DDMmS & 2017 & System & The elderly \\
MEMMHCS & 2019 & Design and Development & General population
\end{tabular}

Table 3 Case of study published from 2008 to 2020 according to its functionality and technology used in the solution

\begin{tabular}{|c|c|c|c|}
\hline Case study & Functioning & Technology & Description \\
\hline MADIP & The monitoring of any vital signs. & $\begin{array}{l}\text { Hospital equipament, } \\
\text { Wearable and Smartphone }\end{array}$ & $\begin{array}{l}\text { A MAS framework that uses medical } \\
\text { equipment for patient monitoring. }\end{array}$ \\
\hline MASRFid & $\begin{array}{l}\text { Blood pressure, blood oxygen and heart } \\
\text { rate monitoring. }\end{array}$ & Wearable & $\begin{array}{l}\text { MAS for healthcare control and for } \\
\text { detecting falls in the elderly. }\end{array}$ \\
\hline MASRHMS & The remote monitoring of any vital signs. & Wearable & $\begin{array}{l}\text { MAS for vital signs remote monitoring } \\
\text { of in home care patients. }\end{array}$ \\
\hline MBES & $\begin{array}{l}\text { Collecting ECG, Breathing rate and } \\
\text { Glucose data. }\end{array}$ & Wearable & $\begin{array}{l}\text { Design of an integrated MAS for sending } \\
\text { and processing medical data intelligently. }\end{array}$ \\
\hline DDMmS & The remote monitoring of any vital signs & Wearable & $\begin{array}{l}\text { MAS for the daily physiological } \\
\text { conditions data analysis of a group from } \\
\text { an elderly people nursing home. }\end{array}$ \\
\hline MEMMHCS & The remote monitoring of any vital signs & $\begin{array}{l}\text { Smartphone / } \\
\text { Wearable }\end{array}$ & $\begin{array}{l}\text { MAS for the remote monitoring of } \\
\text { patients, using Data mining techniques. }\end{array}$ \\
\hline
\end{tabular}

\section{Systems architecture}

As for the architectures, the following parameters were selected from the literature review and are summarized in Tables 4 \& Tables 5. It is important to be emphasized that the proposals were validated through a simulation environment. The proposals based on JADE, MADIP, ${ }^{8}$ MASRHMS ${ }^{9}$ and $\mathrm{DDMmS}^{10}$ used the framework environment itself to carry out their simulations. The proposal that used Aglets, MADIP, ${ }^{8}$ simulated its environment on the Tahiti server, provided by the platform itself. The remaining proposals created their own simulation environment. ${ }^{11,15}$ Intelligent agents can be classified according to their mobility. That way, a MAS can contain static agents ${ }^{8,12}$ or mobile ones, ${ }^{8}$ according to the system need. Interagent communication may or not follow a pre-established protocol or language. Some of the most used protocols and languages in this communication are the: KIF, KQML ${ }^{8}$ FIPA-ACL ${ }^{9,10}$ and XML. When no protocol or language is used, it is called Free Format. ${ }^{6,11}$ In the case of the study MBES, ${ }^{15}$ it makes the use of network protocols for the exchange of messages between MAS agents. In order to facilitate the development of a MAS, several frameworks, platforms and libraries can be used, ${ }^{2}$ such as: Aglets, Cougaar, Fipa-OS, JADE, JATLITE, OAA and Zeus. ${ }^{16}$ The studies reviewed only used three of these as encountered in Aglets, ${ }^{8} \mathrm{JADE}^{8-10}$ and OAA..$^{11,15}$

Table 4 Types of system architecture and its main parameters according to the case of study published

\begin{tabular}{|c|c|c|c|}
\hline Parameters & MADIP & MBES & MASRFid \\
\hline Library / Framework & Aglets & OAA & JADE \\
\hline Privacy and Security & Aglets' Standard Security & Not Informed & JADE's Standard Security \\
\hline MAS Programming Language & JAVA & Not Informed & JAVA \\
\hline Decision Mechanism & $\begin{array}{l}\text { Single Agent Analysis } \\
\text { (Diagnostic Agent) }\end{array}$ & $\begin{array}{l}\text { Distributed Analysis } \\
\text { (Coordination and Consensus } \\
\text { Algorithms) or } \\
\text { Manual Input by the Physician }\end{array}$ & $\begin{array}{l}\text { Distributed Analysis } \\
\text { (Coordination and Consensus } \\
\text { Algorithms) }\end{array}$ \\
\hline
\end{tabular}


Table 5 Types of system architecture and its main parameters according to the case of study published (Cont.)

\begin{tabular}{|c|c|c|c|}
\hline Parameters & MEMMHCS & DDMmS & MASRHMS \\
\hline Simulation Environments & Developed by themselves & JADE Architectural Simulations & JADE Architectural Simulations \\
\hline $\begin{array}{l}\text { Classification of Agents } \\
\text { regarding their mobility }\end{array}$ & Static & Static & Static \\
\hline Library / Framework & OAA & JADE & JADE \\
\hline $\begin{array}{l}\text { Inter-Agent Communication } \\
\text { Technology }\end{array}$ & Free-Format & $\begin{array}{l}\text { Messages } \\
\text { (FIPA - ACL) }\end{array}$ & $\begin{array}{l}\text { Messages } \\
\text { (FIPA - ACL) }\end{array}$ \\
\hline Privacy and Security & Not Informed & JADE's Standard Security & JADE's Standard Security \\
\hline MAS Programming Language & Not Informed & JAVA and $R$ & JAVA \\
\hline Decision Mechanism & $\begin{array}{l}\text { Single Agent } \\
\text { Analysis (Decision } \\
\text { Support Agent) and } \\
\text { Data mining }\end{array}$ & $\begin{array}{l}\text { Distributed Analysis } \\
\text { (Coordination and Consensus } \\
\text { Algorithms) }\end{array}$ & $\begin{array}{l}\text { Distributed Analysis } \\
\text { (Coordination and Consensus } \\
\text { Algorithms) and } \\
\text { Data mining }\end{array}$ \\
\hline
\end{tabular}

\section{Implementation}

In order to maintain the integrity of the data being handled and the MAS integrity, it was noticed that privacy and security mechanisms and policies are required. Some researches made use of mechanisms already included in the $\mathrm{MAS}^{7-10}$ platforms/frameworks/libraries, while others did not mention it. ${ }^{11,15}$ There are several programming languages that can be used for the implementation of intelligent agents and MAS, ${ }^{3,4}$ each containing its own paradigm as shown in Table 6. In order to make the final decision and send the response to the customer (i.e, the one who can be an intelligent agent), it was noticed in this overview that another MAS or a system user is often used by coordination and consensus (Distributed Analysis). ${ }^{2,6}$ In some studies, the role of the leader is assigned to a specific agent, such as the Diagnostic Agent in MADIP $^{8}$ and Decision Support Agent in MEMMHCS. ${ }^{11}$ In some other studies, the use of Datamining algorithms is explored, with the objective of optimizing the leader agent decision MASRHMS ${ }^{9}$ and MEMMHCS, ${ }^{11}$ while in the proposal MBES $^{15}$ the MAS decision can be replaced by a user manual input.

Table 6 Different types of programming languages used in the implementation of intelligent agents

\begin{tabular}{ll}
\hline Programming languages & Paradigm \\
\hline C & $\begin{array}{l}\text { Structured, Imperative and } \\
\text { Procedural } \\
\text { Structured, Imperative and }\end{array}$ \\
C++ & $\begin{array}{l}\text { Procedural, supporting the } \\
\text { object-oriented paradigm }\end{array}$ \\
C\# & Object-Oriented \\
JAVA & Object-Oriented \\
Lisp & Functional \\
Prolog & Declarative \\
R & Functional \\
\hline
\end{tabular}

\section{Discussions}

As a resume, Figure 1 shows the comparison of data collection technologies with the different aspects mentioned. It can be observed that some of the studies reviewed have more than one parameter, therefore, these are counted as a publication in all of the items of which they are a part of. It can also be observed that Wearable technology was the most used one, followed by Smartphones, which leads us to believe that the concern with remote care and the use of technologies combined with Internet of Things have been growing in the academia. Regard to the technology and its implementation, most of these type of studies uses Distributed Analysis as a decision mechanism. This is due to the fact that the distributed analysis is part of the distributed artificial intelligence, whose analysis can provide fault tolerance, higher security for the system and reliability for the decision maker. Looking at the architecture characteristic, static agents predominate as the type of agents used in MAS, while the most used MAS development platform is JADE. It is important to mention that the JADE platform is a framework for implementing static smart agents and supports agent mobility, then it has been one of the most updated MAS platforms with the largest community participation.

Medical care application using MAS and Wearable are the main targets if the objective is to obtain a good Data Analysis. It was found a few number of researches which present a ready solution type of the implementing system. We believe that this is due to the fact that implementing a system takes much longer and requires a lot of knowledge in programming languages and software engineering, in addition to the fact that its implementation is necessary to validate any proposal for this kind. Most of the studies focused more on presenting solutions using Smartphones. Only one article considered the use of Medical Equipment, thus it seems that there is lack of study in this area. Since hospital equipment cannot be excluded from a patient's life, whether being monitored or not, this absence might result for the increasing number of advanced remote monitoring systems and, at the same time, for the obsolescence of hospital equipment. It was found many different types of aspects and its respective objectives at each research paper, as shown in Figure 2. Some of the studies reviewed have more than one parameter, therefore, these are counted as a publication in all of the items of which they are a part of it. It can be observed that the Design occupies the largest share of studies followed by the Development, which might be the fact that the proposal of a system architecture is much less expensive and demands less time than implementing a system. The proposals for Systems already implemented take the smallest slice of it, showing a deficit for such solutions. The implementation of a system requires not only its conception, but a high knowledge in software engineering, 
frameworks and programming languages, and much more time for its final implementation. In terms of programming languages, JAVA is the most used one due to its facility as a programming language and because the JADE platform is designed in JAVA.

If we consider the Development Platforms for Design, OAA occupies the largest share, followed by the JADE platform and the Aglets library. Since future implementations in a system might take place, thus leaving the platform open is highly recommended due to the fact that novel frameworks may appear to be better than the current ones. It was also noticed that JADE is the most used implementation tool for a healthcare system, because it is considered to be the most updated agent framework and to have an easy communication channel to the project developer. In terms of the mobility of MAS agents, static agents are the majority for both Design and Development, since the need for implementation of mobile agents in a system requires a careful study when building the system setup. The last depends on the machine infrastructure and permissions of the network. As showed in Figure 1, Wearable technology is the most used one for both Design and Development, followed by Smartphones. Due to the increasing number of worldwide population needing hospitals and clinics, specially the elderly one, bedridden patients and the general public are the most researched target population in the Design objective, while the elderly one is studied the most in regards to the System objective.

\section{Conclusions}

This research presented a systematic review on intelligent agents and MAS in biomedical engineering. Due to the short time for this research due to the COVID-19 Pandemic, only six studies were selected from the databases described in the methodology. The keyword that presented the most consistent result for the purpose of this review was «health monitoring» multi-agent system. The other ones used in the search often returned many publications. There are a large number of studies using MAS in healthcare with different purposes other than those desired for this review, such as coordinating ambulances, ${ }^{17}$ interaction between hospitals ${ }^{18}$ and the optimization of hospital data. ${ }^{19-23}$ It is also worth mentioning that some keywords returned results not only for the full keyword but for part of it, such as Multi Agent ${ }^{24}$ and Health. ${ }^{25}$ It was observed that most of the proposals that address the topic were found in the IEEE database, while the $\mathrm{ACM}$ one presented just few search results that trully report the use of intelligent agents and MAS in biomedical engineering.

It can be concluded taht there was a deficit in researches which might be used as ready-to-use system. Most of the proposals aim to contribute merely to the design and further developments. In addition, it was also noticed that only one contribution was concerned with the implementation or extension of MAS for its use in medical equipment together with wearables and smartphones. It must be pointed out the fact that there is a lack of a complete data set and parameters in most of the studies reviewed here, either not mentioning the programming language used nor the type of privacy and security policy. This lack of information may raise doubts whether the proposed MAS is really safe and how far the work would be heterogeneous or not. The more the technical characteristisc are describeb in a rearche paper the better will be the output of such literature review and, consequently, more clear specific issues will be presented in order to improve and guide wordwide technologies in the healthcare comummity. ${ }^{26}$

\section{Acknowledgments}

We thank the State University of Santa Catarina (UDESC) for the institutional support and the State Research Foundation of Santa Catarina (FAPESC) for the financial support.

\section{Conflicts of interest}

The authors declare that there is no conflict of interest.

\section{References}

1. Filgueiras TP, Leonardo MR, Luciana de Oliveira Rech, et al. RTJADE: A preemptive real-time scheduling middleware for mobile agents. Concurrency and Computation: Practice and Experience. 2018;31(13):e5061.

2. Kravari K, Bassiliades N. A survey of agent platforms. Journal of Artificial Societies and Social Simulation. 2015;18(1):11.

3. Larsen JB. Agent programming languages and logics in agent-based simulation. Modern approaches for intelligent information and database systems. 2018:517-526.

4. Payam Porkar Rezaeiye, Pasha Porker Rezaeiye, Esmaeil Fakhimi Gheshlagh Mohammad Beig, et al. Agent programming with object oriented (C++). 2017 Second International Conference on Electrical, Computer and Communication Technologies (ICECCT). Coimbatore. 2017:1-10.

5. Leppänen T, José Álvarez Lacasia, Yoshito Tobe, et al. Mobile crowdsensing with mobile agents. Autonomous Agents and Multi-Agent Systems. 2017;31(1):1-35.

6. Iqbal S, Wasif Altaf, Muhammad Aslam, et al. Application of intelligent agents in health-care. Artificial Intelligence Review. 2016;46(1):83-112.

7. SUH, Shih SC. Building Distributed E-Healthcare for Elderly Using RFID and Multi-Agent. International Journal of Engineering Business Management. 2011;3(1):16-26.

8. Su J. Mobile multi-agent based, distributed information platform (MADIP) for wide-area e-health monitoring. Computers in industry. 2008;59(1):55-68.

9. Abraham, Hela Ltifi, Mounir Ben Ayed, et al. Multi-agent Architecture for Visual Intelligent Remote Healthcare Monitoring System. Hybrid Intelligent Systems, Advances in Intelligent Systems and Computing. 2016.

10. Solange Mendes, Jonas Queiroz, Paulo Leitão, et al. Data driven multiagent $\mathrm{m}$-health system to characterize the daily activities of elderly people. 2017 12th Iberian Conference on Information Systems and Technologies (CISTI). IEEE. 2017.

11. Polu SK. Modeling of Efficient Multi-Agent based Mobile Health Care System. International Journal for Innovative Research in Science and Technology. 2019(5).

12. ACM. Association for Computing Machinery. Advancing Computing as a Science and Profession.

13. IEEE. IEEE - The World's Largest Technical Professional Organization Dedicated To Advancing Technology For The Benefit Of Humanity.

14. Science direct. Explore scientific, technical, and medical research on ScienceDirect.

15. Toader CG. Multi-agent based e-health system. In: 201721 st International Conference on Control Systems and Computer Science. 2017.

16. OAA. The Open Agent Architecture. 2020.

17. Beatriz López, Bianca Innocenti, Dídac Busquets, et al. A multiagent system for coordinating ambulances for emergency medical services. IEEE Intelligent Systems. 2008:23(5):50-57.

18. Hanen Jemal, Zied Kechaou, Mounir Ben Ayed, et al. Swarm intelligence and multi agent system in healthcare. 2014 6th International Conference of Soft Computing and Pattern Recognition (SoCPaR). IEE. 2014.

19. Vieira-Marques P, Patriarca-Almeida J, Frade S, et al. OpenEHR aware multi agent system for inter-institutional health data integration. 2014 9th Iberian Conference on Information Systems and Technologies (CISTI). IEEE. 2014. 
20. Sara Rodríguez, Carolina Zato, Corchado JM, et al. Fusion system based on multi-agent systems to merge data from WSN. 17th International Conference on Information Fusion (FUSION). Salamanca. 2014:1-8.

21. Jemal H, Zied Kechaou, Mounir Ben Ayed, et al. Cloud computing and mobile devices based system for healthcare application. 2015 IEEE International Symposium on Technology and Society (ISTAS). Dublin. 2015:1-5.

22. Henry Okoyo, Kevin Mugoye, Sylvester Mcoyowo, et al. Smart-bot Technology: Conversational Agents Role in Maternal Healthcare Support. 2019 IST-Africa Week Conference (IST-Africa), Nairobi, Kenya. 2019:17.

23. J Wu, Shenfang Yuan, Sai Ji, et al. Multi-agent system design and evaluation for collaborative wireless sensor network in large structure health monitoring. Expert Systems with Applications. 2010;37:20282036.
24. Gupta M, Bertrand JW, Sabarish V Babu, et al. An evolving multi-agent scenario generation framework for simulations in preventive medicine education. Proceedings of the 2nd ACM SIGHIT International Health Informatics Symposium. 2012.

25. Regien G Biesma, Ruairí Brugha, Andrew Harmer, et al. The effects of global health initiatives on country health systems: a review of the evidence from HIV/AIDS control. Health policy and planning. 2009;24(4):239-252.

26. Mouratidis H, Paolo G, Manson G, et al. Modelling secure multiagent systems. In Proceedings of the second international joint conference on Autonomous agents and multiagent systems (AAMAS '03). Association for Computing Machinery, New York, NY, USA. 2003:859-866. 